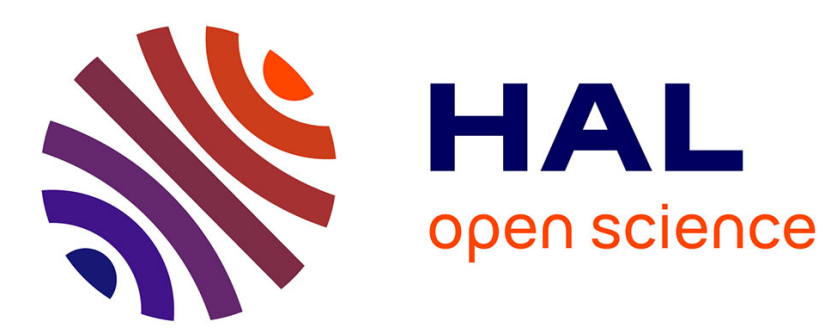

\title{
Etude in situ du fonctionnement hydrique et photosynthétique d'une vigne conduite en lyre
}

Nader Katerji, François-Alain Daudet, Brigitte Durand, René Pallut, Annie Duparque

\section{- To cite this version:}

Nader Katerji, François-Alain Daudet, Brigitte Durand, René Pallut, Annie Duparque. Etude in situ du fonctionnement hydrique et photosynthétique d'une vigne conduite en lyre. Agronomie, 1986, 6 (8), pp.709-716. hal-00884928

\section{HAL Id: hal-00884928 https://hal.science/hal-00884928}

Submitted on 1 Jan 1986

HAL is a multi-disciplinary open access archive for the deposit and dissemination of scientific research documents, whether they are published or not. The documents may come from teaching and research institutions in France or abroad, or from public or private research centers.
L'archive ouverte pluridisciplinaire HAL, est destinée au dépôt et à la diffusion de documents scientifiques de niveau recherche, publiés ou non, émanant des établissements d'enseignement et de recherche français ou étrangers, des laboratoires publics ou privés. 


\title{
Etude in situ du fonctionnement hydrique et photosynthétique d'une vigne conduite en lyre
}

\author{
Nader KATERJI \& François-Alain DAUDET $\left(^{*}\right)$ \\ avec la collaboration technique de Brigitte DuRand, René Pallut \& Annie Duparque \\ I.N.R.A., Station de Bioclimatologie, Centre de Recherches de Paris-Grignon, F 78850 Thiverval-Grignon \\ (*) I.N.R.A., Laboratoire de Bioclimatologie, Domaine de Crouelle, F 63039 Clermont-Ferrand
}

RÉSUMÉ

Le fonctionnement hydrique et photosynthétique d'une vigne a été suivi en milieu naturel au cours de plusieurs journées à l'aide de mesures de résistance stomatique, du potentiel hydrique foliaire et des échanges de $\mathrm{CO}_{2}$. L'influence de la réhumectation du sol a pu être étudiée à l'échelle du cep entier. Par ailleurs, les études poursuivies sur les bras opposés d'un même cep ont permis de mettre en évidence des différences de comportement qui semblent en liaison avec le microclimat lumineux de ces bras.

Mots clés additionnels : Assimilation nette, résistance stomatique, potentiel hydrique foliaire.

In situ study of the water and photosynthetic functions of a lyre-shaped grapevine.

The water and photosynthetic functions of a grapevine were observed for several days under field conditions by measuring stomatal resistance, leaf water potential and $\mathrm{CO}_{2}$ exchange. The influence of soil humidification was studied on the whole plant. In addition, studies carried out on opposite branches of the same vine showed behavioral differences which seem linked to the light microclimate of these branches.

Additional key words : Leaf water potential, stomatal resistance, net photosynthesis.

\section{INTRODUCTION}

Les études concernant l'influence des principaux paramètres climatiques sur la vigne remontent à une centaine d'années (voir l'historique de CARBONNEAU, 1980). Ces premiers travaux avaient une vocation agroclimatique (FOEX, 1895 ; RAVAZ, 1909) ; en particulier, ils étaient orientés vers la définition des limites géographiques de la vigne et de ses exigences en lumière, température et sécheresse vis-à-vis de la production de vins de qualité. Beaucoup plus tard, et avec le développement des moyens techniques adéquats, on s'est orienté vers des analyses plus fines touchant notamment à l'influence du microclimat parcellaire sur le fonctionnement de la vigne. Deux axes principaux de recherche se dégagent :

- le $1^{\text {er }}$ concerne l'étude des relations entre les conditions pédoclimatiques (eau, lumière, température) et la production finale (KRIEDEMANN, 1968 ; SEgUiN, 1970 ; KLIEWER \& LIDER, 1970 ; TSEHORNG-LIU, 1974) en intégrant comme paramètres de variation des facteurs propres à la végétation : effet variétal, stade de développement, âge... (RIBEREAU-
GAYON, 1966 ; KRIEDEMANN et al., 1970 ; KRIEDEMANN et al., 1973) ou de phytotechnie (SHAULIS et al., 1966 ; MILLAR, 1972) ;

- le $2^{\mathrm{e}}$, plus récent, porte sur l'étude des fonctionnements photosynthétique et hydrique de la plante à une échelle de temps plus fine. Cette orientation, favorisée aujourd'hui par le perfectionnement des techniques écophysiologiques, tend à accompagner le développement et la recherche de nouvelles pratiques phytotechniques (taille, modes de conduite, ...) répondant à la fois aux nécessités d'une mécanisation accrue et d'une amélioration des qualités œnologiques.

SMART $(1973,1976)$ a clairement montré que le système de conduite peut modifier le microclimat lumineux du feuillage et influer fortement sur la production de raisin. A partir d'un modèle décrivant la répartition horaire de l'éclairement en fonction de l'écartement, de la hauteur et de l'orientation des plans de feuillage, et en adoptant le critère d'une captation maximale de l'énergie lumineuse, cet auteur a souligné l'intérêt des orientations nord-sud et des vignes « ouvertes » (plantations larges). 
Carbonneau (1980) puis Carbonneau \& DE LOTH (1985) ont étudié, à partir du modèle précédent, l'effet sur l'interception du rayonnement solaire direct de diverses combinaisons d'orientations et d'inclinaisons de plans de feuillage. Les conclusions de leur étude théorique ont été confrontées avec des mesures ponctuelles de résistance stomatique et de photosynthèse brute. Il semble ressortir de ces $1^{\text {res }}$ mesures que l'augmentation de l'énergie solaire captée au cours de la journée peut s'accompagner d'une diminution des échanges gazeux par suite d'un accroissement des résistances stomatiques et/ou des « résistances internes " à la fixation photosynthétique du $\mathrm{CO}_{2}$. On ne sait pas à l'heure actuelle l'origine précise de ces phénomènes (contrainte hydrique, concentration en assimilats...), par ailleurs maintes fois constatés chez diverses cultures.

Ainsi, augmentation de l'énergie lumineuse interceptée et augmentation de la photosynthèse ne vontelles pas forcément de pair. Par ailleurs, les contraintes subies en cours de journée, si elles ont indubitablement un effet dépressif sur la quantité d'assimilats produits, peuvent avoir des conséquences positives sur l'élaboration des critères qualitatifs.

Ces quelques réflexions situent l'apport actuel de travaux écophysiologiques du type de ceux que nous présentons ci-dessous et que l'on peut rattacher au $2^{\mathrm{e}}$ des axes de recherche que nous venons de définir. Deux problèmes seront abordés :

- le fonctionnement hydrique et photosynthétique à l'échelle du cep de vigne,

- les différences de comportement observées entre 2 bras opposés d'un même cep.

Plusieurs hypothèses seront avancées pour interpréter les résultats obtenus.

\section{MATÉRIEL ET MÉTHODES}

Les résultats que nous présentons ci-dessous ont été obtenus au cours d'une campagne de mesure de $12 \mathrm{j}$ (18-30 août 1984) au Domaine INRA du Grand Parc à Latresne (Gironde), dans la zone d'appellation « Premières côtes de Bordeaux ».

\section{A. Matériel végétal et mode de conduite}

Le matériel végétal est du Cabernet-Sauvignon greffé sur 101/14, planté en 1976 avec conduite en "lyre ». Ce mode de conduite (CARBOnNEAU, 1980) est directement issu d'une réflexion sur l'efficience de la captation de la lumière par les vignes. Il consiste à palisser les ceps de telle sorte que 2 bras maîtres soient formés perpendiculairement au rang. Les ramifications secondaires portées par chacun de ces bras sont attachées sur des fils de fer tendus de part et d'autre du rang sur des supports en bois disposés en $\mathrm{V}$ mais les ramifications les plus longues ont tendance à retomber librement dans l'inter-rang donnant à l'ensemble un profil en lyre caractéristique (fig. 1). La surface captatrice de lumière est ainsi divisée en plusieurs plans d'inclinaisons différentes et elle se trouve largement ouverte vers le haut. Compte tenu de l'orientation nord-sud des rangs de la vigne étudiée

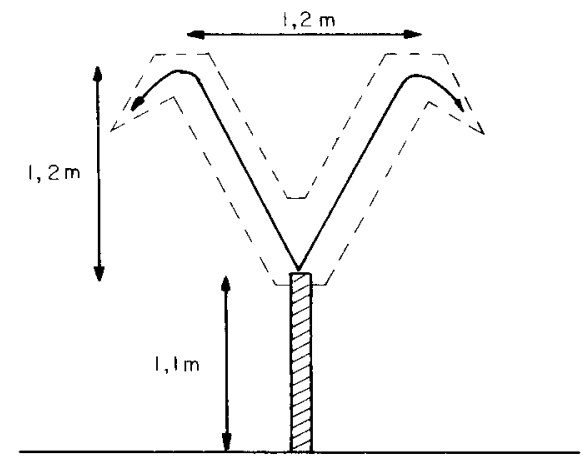

Figure 1

Coupe transversale schématique d'une vigne conduite en lyre. (D'après CARBONNEAU, 1978). Diagrammatic cross section of a grape vine grown in lyre shape (from CARBONNEAU, 1978).

WTith Tronc, trunk; ___ branches ; - . - volume de feuillage, volume of foliage.

ici, nous emploierons dans la suite, les termes de " capteur est " ou " ouest » pour désigner l'ensemble des rameaux et feuilles issus de chaque bras maître. Nous serons également amenés à distinguer sur ces capteurs les surfaces inférieure et supérieure qui se trouvent dans des conditions d'éclairement très différentes.

Cinq individus voisins d'une même ligne, constituant un "lot homogène " (ce qui signifie ici que la variabilité individuelle n'est pas étudiée et qu'en particulier les mesures destructives sont effectuées sur des échantillons de feuilles prélevées sur ces 5 ceps), ont été retenus. Ils sont référencés par leur rang de 1 à 5 .

\section{B. Milieu pédoclimatique}

\section{Le sol et les conditions d'enracinement}

Constitué d'une grave sablo-limoneuse sur au moins $1,80 \mathrm{~m}$ de profondeur, le sol est caractéristique de beaucoup de terrains réservés à la vigne. Il ressort de l'étude des profils hydriques que l'alimentation en eau des vignes semble se faire difficilement en dessous de $1,20 \mathrm{~m}$ de profondeur (KATERJl et al., 1986).

\section{Le climat}

Le climat, de type océanique, est caractérisé par des températures estivales quelquefois élevées.

Les données climatiques journalières sont recueillies par une station météorologique automatique située sur le domaine. Le tableau 1 les présente pour la période d'étude. Les 3 premières journées, qui sont extrêmement chaudes, font suite à une longue période sans pluie. Les journées suivantes sont au contraire assez pluvieuses (orages) et nettement moins chaudes.

Les données instantanées de rayonnement global, température de l'air et hygrométrie au cours des mesures rapportées ici ont été fournies par des capteurs associés au système de mesure des échanges gazeux (cf. II.C.4).

\section{Méthodes et techniques employées}

\section{Mesure de la conductance stomatique $H$}

Les mesures de conductance stomatique $\mathbf{H}$ (inverse de la résistance stomatique $r s$ ), réalisées de façon 
TABLEAU 1

Données climatologiques journalières fournies par la station automatique au cours de la période du 18 au 30 août 1984. Daily climatological data provided by the automatic station during the period August 18-30, 1984.

\begin{tabular}{lccccccccccccc}
\hline \multicolumn{1}{c}{ Date } & 18 & 19 & 20 & 21 & 22 & 23 & 24 & 25 & 26 & 27 & 28 & 29 & 30 \\
\hline T. $\min \left({ }^{\circ} \mathrm{C}\right)$ & 16,6 & 16,0 & 16,6 & 16,6 & 16,2 & 15,6 & 15,9 & 15,7 & 14,1 & 16,0 & 15,4 & - & 16,5 \\
T. $\max \left({ }^{\circ} \mathrm{C}\right)$ & 31,8 & 34,0 & 32,2 & 28,0 & 25,5 & 24,1 & 22,0 & 24,4 & 24,9 & 24,8 & 26,9 & - & 28,9 \\
R.G. $\left(\mathrm{J} . \mathrm{cm}^{-2} \cdot \mathrm{j}^{-1}\right)$ & 220 & 2411 & 2040 & 1126 & 1145 & 1388 & 1303 & 1780 & 1505 & 1509 & 1783 & - & 2050 \\
1,0 & 0 & 0 \\
Pluie $(\mathrm{mm})$ & 0 & 0 & 0 & 11,4 & 1,0 & 3,4 & 43,1 & 5,8 & 0,8 & 1,0 & 0 \\
\hline \hline
\end{tabular}

horaire, à l'aide d'un poromètre à diffusion de vapeur d'eau (Automatic Porometer MK II) ont porté sur la face inférieure des feuilles (la face supérieure des feuilles de vigne est dépourvue de stomates). Lors de chaque mesure, des feuilles bien dégagées sont choisies dans la strate supérieure de la végétation. Le poromètre est reétalonné assez fréquemment pour corriger l'effet des variations de la température ambiante. La conductance moyenne est évaluée par la moyenne arithmétique de 10 conductances ponctuelles mesurées dans chaque situation.

\section{Mesure du potentiel hydrique foliaire $\psi_{F}$}

Des mesures horaires de potentiel foliaire sont également effectuées sur des feuilles placées dans la même situation (bien éclairées, strate supérieure). Six feuilles sont prélevées dans chaque situation étudiée et leur potentiel est immédiatement mesuré dans une chambre de pression (SCHOLANDER et al., 1965).

\section{Mesure de la surface foliaire}

La surface foliaire totale des ceps a été déterminée par A. CARBOnNeau sur les individus 3 et 5 suivis du point de vue des échanges gazeux. La méthode non destructive qu'il emploie a été précédemment décrite (CARBONNEAU, 1976a). L'erreur est estimée à environ 5 p. 100 (CARBONNEAU, 1976b).

\section{Mesure des échanges gazeux}

Nous avons utilisé une Chambre d'Assimilation et de Transpiration (CAT) décrite par DAUDET (1986). Il s'agit d'un système fonctionnant en circuit ouvert (avec un léger enrichissement en $\mathrm{CO}_{2}$ ), destiné à l'étude in situ d'échantillons végétaux relativement volumineux ; le débit général d'air traversant la chambre d'assimilation (enceinte en film polyéthylène mince), de l'ordre de 3 à $4 \mathrm{~m}^{3} . \mathrm{mn}^{-1}$, assure un taux de renouvellement suffisamment élevé pour minimiser les effets de serre. Dans le cas présent, à cause des fils de palissage de la vigne (qui sont restés en place), l'enceinte a dû être munie d'une fermeture spéciale (VELCRO + ruban adhésif double face).

Outre les échanges gazeux (assimilation nette et transpiration) ce système mesure en continu le rayonnement global incident (pile solarimétrique de Kipp) ainsi que la température et l'humidité de l'air à l'entrée et à la sortie de l'enceinte. Après un calcul des moyennes sur des périodes d'environ $3 \mathrm{mn}$ les données sont stockées sur microcassette magnétique. Dans la plupart des cas nous présenterons cependant, par souci de lisibilité, des résultats correspondant à un regroupement d'un nombre plus ou moins important de ces périodes élémentaires.

Les ceps de rang 5 et 3 de l'échantillon ont successivement été étudiés. Leur feuillage était soit entièrement placé dans l'enceinte (étude de la lyre entière) soit par moitié (étude de demi-lyre est ou demi-lyre ouest). Le volume de l'enceinte pour la lyre entière était approximativement de $1 \mathrm{~m}^{3}$ alors qu'il n'était que de $0,3 \mathrm{~m}^{3}$ pour les demi-lyres. Dans les 2 cas, le débit général de l'air traversant l'enceinte était de l'ordre de $3 \mathrm{~m}^{3} \cdot \mathrm{mn}^{-1}$.

\section{RÉSULTATS}

\section{A. Fonctionnement de la lyre entière}

La figure $2(a, b, c)$ montre l'évolution du rayonnement global, de la température de l'air et de l'assimilation nette d'une lyre entière pour les journées du 19 et du 22 août. Ces journées correspondent à une situation de sol sec avec un temps très ensoleillé et très chaud pour la première, à un début de réhumectation du sol (lendemain d'une pluie de $11,4 \mathrm{~mm}$ ) et à un temps variable et doux pour la seconde.

La figure 3 ( $a$ et $b$ ) donne l'évolution des valeurs moyennes de conductance stomatique et de potentiel hydrique foliaire pour les mêmes journées.

Les ceps étudiés présentent des "vigueurs " voisines au moins si l'on en juge par les surfaces foliaires peu différentes : $5,42 \mathrm{~m}^{2}$ pour le cep $\mathrm{n}^{\circ} 5$ étudié le 19 août contre $5,76 \mathrm{~m}^{2}$ pour le cep $\mathrm{n}^{\circ} 3$ étudié à partir du 21 août. L'évolution très contrastée de l'assimilation nette entre les 2 journées s'explique vraisemblablement par les différences d'état hydrique des plantes et de température :

- Le 19 août, le maximum d'assimilation nette est observé à 7 h 30 (TU) alors que le rayonnement global est inférieur à $300 \mathrm{~W} \cdot \mathrm{m}^{-2}$. Par ailleurs, dès midi TU, le bilan de la fixation de $\mathrm{CO}_{2}$ devient nul ou légèrement négatif. Le potentiel hydrique foliaire qui vaut déjà $-0,7 \mathrm{MPa}$ peu de temps après le lever du soleil (potentiel de base), devient inférieur à $-1,1 \mathrm{MPa}$ à partir de $7 \mathrm{~h} 30$ et atteint des valeurs très proches de - 1,5 MPa en milieu de journée. La conductance stomatique prend rapidement des valeurs très faibles.

- Le 22 août, l'assimilation nette suit l'évolution du rayonnement global. Le potentiel hydrique de base est d'environ - 0,2 $\mathrm{MPa}$ alors que le minimum, atteint vers 10-12 h TU est de l'ordre de $-1,1 \mathrm{MPa}$. 


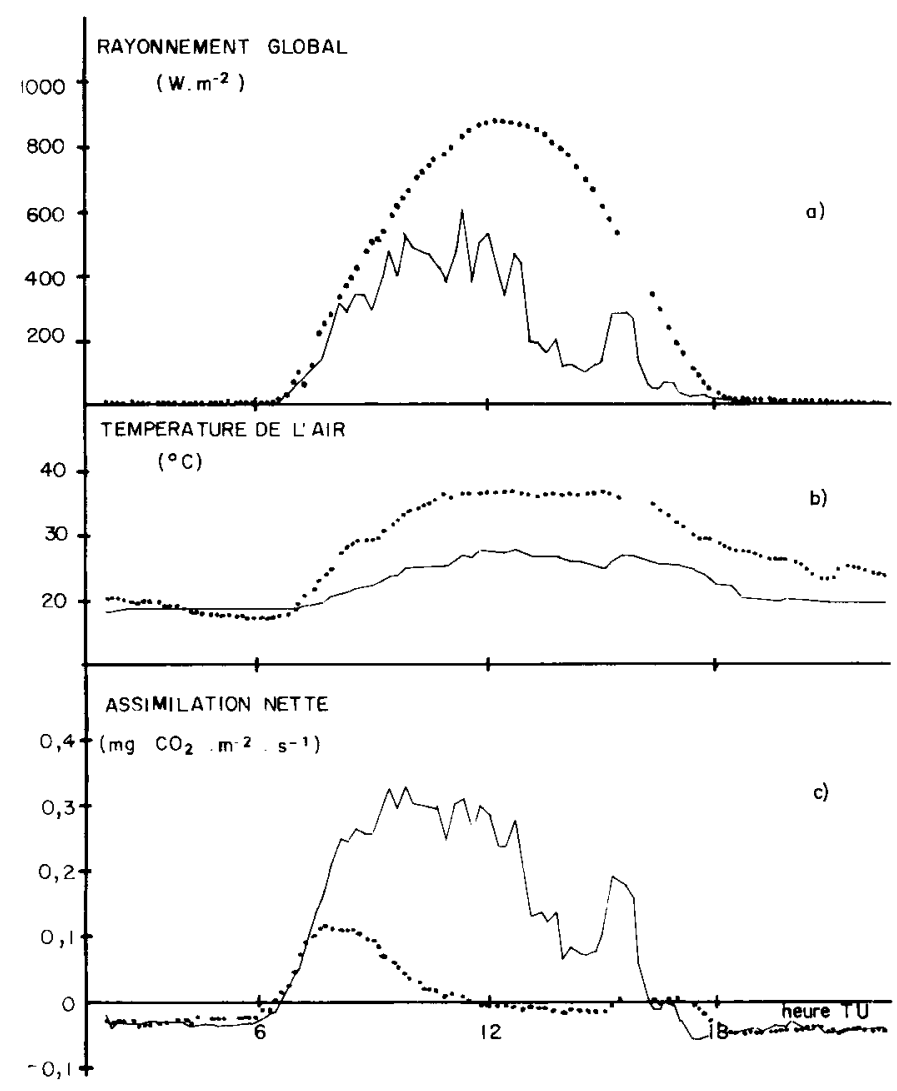

Figure 2

Evolution au cours de 2 journées de : Change over two days in:

a) rayonnement global; total radiation,

b) température de l'air; air temperature,

c) assimilation nette par unité de surface foliaire d'un cep entier ; net photosynthesis per unit of leaf area of an entire vine plant.

-.- journée du 19 août 1984, journée du 22 aô̂t 1984.
Par ailleurs, la conductance stomatique diminue. peu entre le matin et l'après-midi.

La différence entre les 2 journées apparaît aussi sur la figure 4 où sont portées les relations assimilation nette et rayonnement global : pour le 19 août, la figure présente la forme caractéristique des situations avec contraintes - thermique et hydrique - importantes et d'intensité croissante au cours de la journée. Le 22 août, des effets de contrainte existent vraisemblablement aussi à en juger par la légère différence de relation entre matin et soir, mais ils sont peu marqués.

L'optimum thermique pour l'assimilation nette de la vigne est situé, selon les auteurs, entre 25 et $30^{\circ} \mathrm{C}$ (KriedemanN, 1968) ou 22 et $30^{\circ} \mathrm{C}$ (KLIEWER \& LIDER, 1970), or on dépasse $30^{\circ} \mathrm{C}$ pendant la majeure partie du 19 août. La limitation observée est donc probablement due à l'effet conjugué des 2 facteurs : sécheresse et température excessive.

\section{B. Fonctionnement comparé des capteurs Est et Ouest au cours de la journée}

\section{Rappel sur l'éclairement des plans de feuillage}

La figure 5 montre, pour le 20 août et la latitude de Bordeaux, l'évolution théorique de l'éclairement dû au rayonnement solaire direct, de plans parallèles aux « capteurs » définis précédemment : les 2 plans considérés, orientés nord-sud sont inclinés à 72 degrés sur l'horizontale l'un vers l'est l'autre vers l'ouest.

En l'absence de mesures directes d'éclairement parallèlement aux plans du feuillage et d'informations suffisantes pour calculer l'ombrage réciproque des 2 demi-lyres l'une sur l'autre, la seule référence que nous considèrerons est le rayonnement global RG que l'on pourra comparer à l'éclairement théorique par rayonnement direct d'un plan horizontal (fig. 5). Nous n'avons pas, faute d'éléments suffisants, essayé de recalculer, même approximativement à partir de RG, l'éclairement énergétique des différents plans du feuillage.

On remarquera simplement la symétrie, par rapport au midi solaire, de l'évolution de l'éclairement des 2 capteurs considérés. Cette symétrie qui est due à l'orientation nord-sud des rangs conduit à faire l'hypothèse que, dans le cas d'une répartition ellemême symétrique de RG au cours de la journée, les 2 demi-lyres auront subi une irradiation identique.
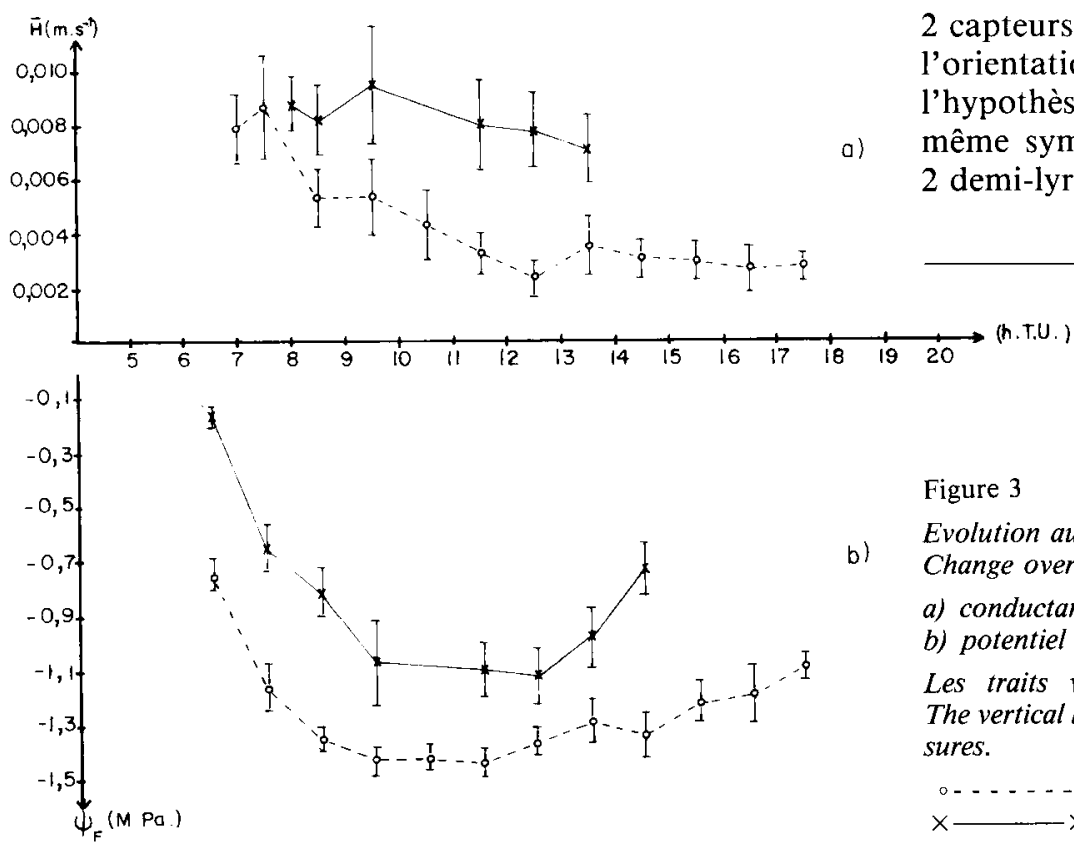

Figure 3

Evolution au cours de 2 journées de:

Change over two days in :

a) conductance stomatique, $H$; stomatal conductance, $H$, b) potentiel hydrique foliaire, $\psi_{F}$; leaf water potential, $\psi_{F}$

Les traits verticaux correspondent à l'écart-type des mesures. The vertical lines correspond to the standard deviation for these measures. 


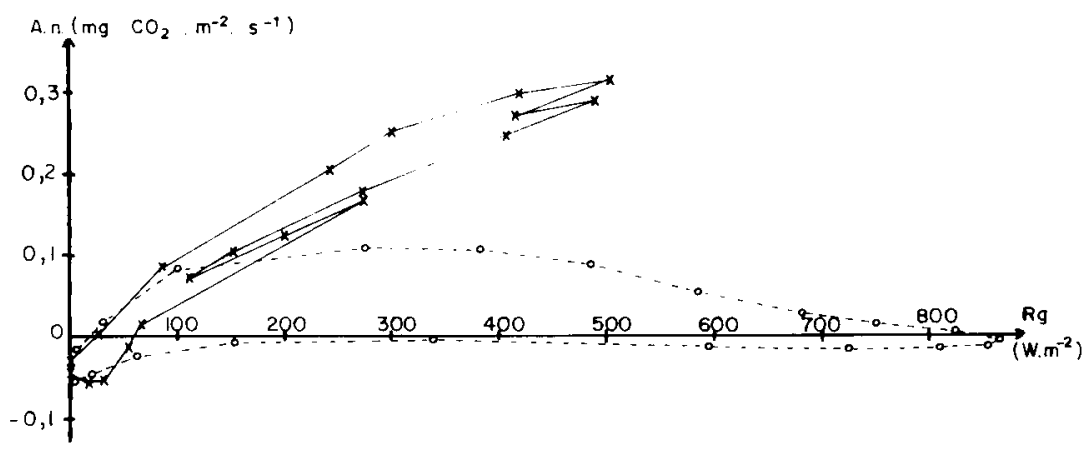

Figure 4

Relation entre assimilation nette d'un cep et rayonnement global au cours de 2 journées.

Relation between net photosynthesis of vine and total radiation over two days.

०-..- - journée du 19 août 1984,

$\times \longrightarrow$ journée du 22 août 1984.

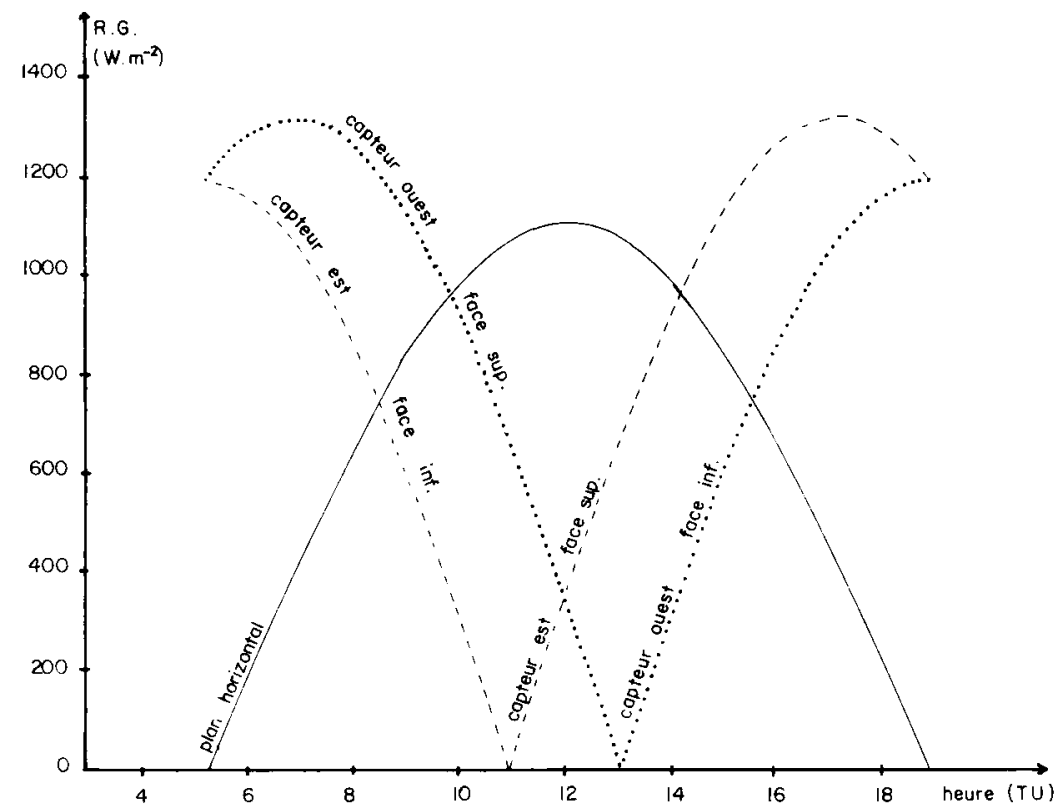

Figure 5

Evolution journalière théorique de l'éclairement de différents plans par le rayonnement solaire direct (Bordeaux, 23 aout); theoretical daily change in illumination of different planes by direct solar radiation (Bordeaux, 23 August).

plan horizontal; horizontal plane.

plan Nord Sud incliné de 72 degrés (capteur est), faces sup. et inf. ; North-South plane slanted, 72 degrees (east branch, upper and lower surfaces),

-... plan Nord Sud incliné de 108 degrés (capteur ouest), faces sup. et inf.; North-South plane slanted 108 degrees (west branch), upper and lower surfaces).

\section{Fonctionnement hydrique}

Sur les figures 6 et 7 , nous avons présenté les valeurs de $\Psi_{\mathrm{F}}$ et $\mathrm{H}$ mesurées simultanément toutes les heures sur la face inférieure des capteurs est et ouest pendant 2 journées successives ( 27 et 28 août).

En ce qui concerne l'évolution de la conductance stomatique $\mathbf{H}$, un décalage apparaît entre les capteurs est et ouest : l'ouverture stomatique maximale sur le bras est, est atteinte à 10-11 h alors qu'on l'observe vers 13-14 h pour le bras ouest ; sur ce dernier, la fermeture stomatique est nettement plus tardive. On note également que la valeur maximale de conductance stomatique est plus élevée sur le capteur est que sur le capteur ouest.

Le même décalage apparaît lorsqu'on compare les 


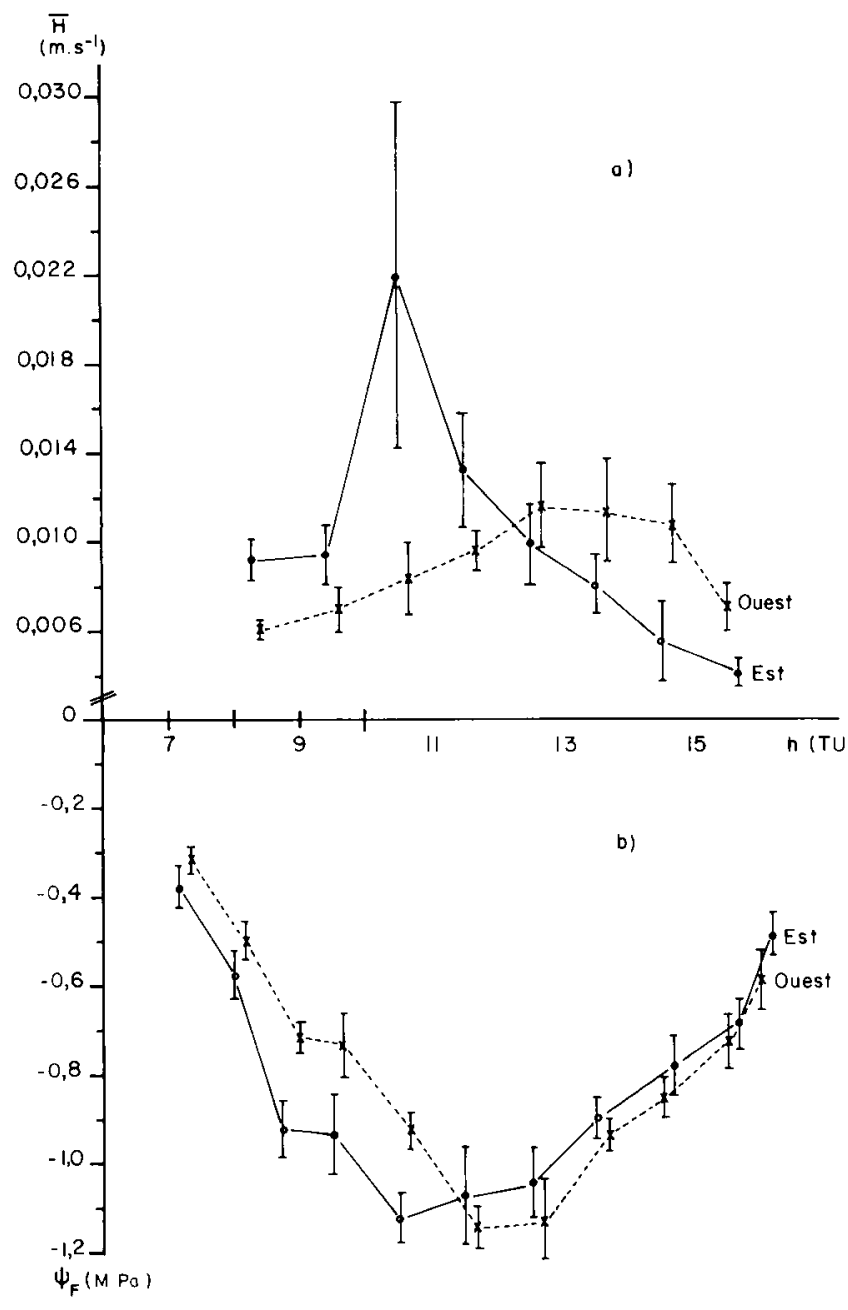

Figure 6

Evolution au cours de la journée (27 août 1984) pour 2 demi-lyres de; Daily course (27 August 1984), for two half-lyres, of :

a) conductance stomatique, $H$; stomatal conductance,

b) potentiel hydrique foliaire, $\psi_{F}$; leaf water potential.

Les traits verticaux représentent l'intervalle de confiance à $95 \%$. The vertical lines represent $5 \%$ standard errors.

cinétiques journalières de potentiel foliaire $\Psi_{F}$ entre l'est et l'ouest. Sur le bras est, le dessèchement foliaire maximal est atteint à 10-11 h alors qu'on ne l'observe sur le bras ouest que 2 à $3 \mathrm{~h}$ plus tard. On note aussi que la valeur minimale de $\Psi_{\mathrm{F}}$ observée sur le capteur ouest est plus basse que celle observée sur le capteur est.

\section{Assimilation nette}

Cette comparaison a pu être faite sur le cep 3 à partir des journées suivantes :

- le 27 août (demi-lyre est),

- les 28,29 et 30 août (demi-lyre ouest).

On comparera également ces mesures à celles effectuées le 25 et le 26 août pour la lyre entière sur le même cep.

Remarquons que toute cette période suit la forte pluie orageuse du 24 août et que, par conséquent, l'on peut admettre que les contraintes hydriques, si elles existent, sont minimales.

Les 2 demi-lyres ont des surfaces foliaires assez différentes :

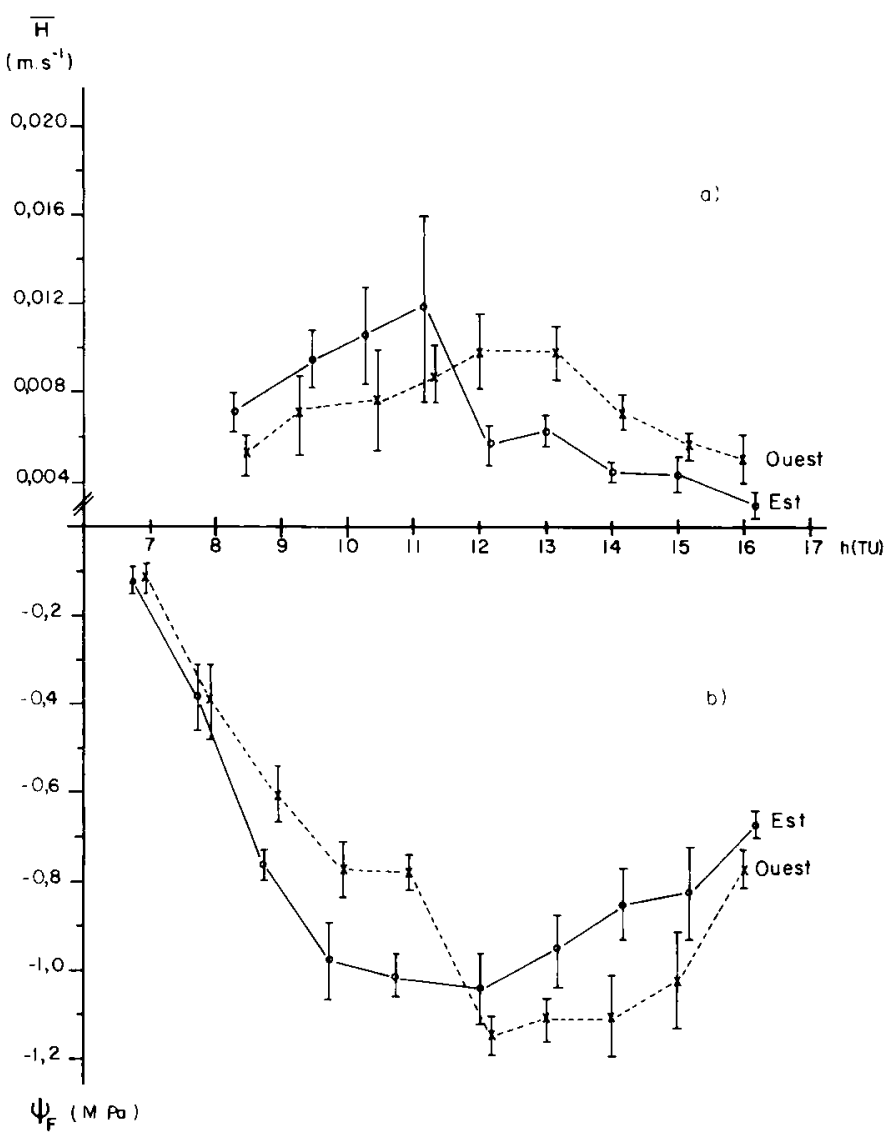

Figure 7

Evolution au cours de la journée (28 août 1984) pour 2 demi-lyres de; Daily course (28 August 1984), for two half-lyres, of:

a) conductance stomatique; stomatal conductance, $H$, b) potentiel hydrique foliaire, leaf water potential, $\psi_{F}$.

Les traits verticaux représentent l'intervalle de confiance à $95 \%$. The vertical lines represent $5 \%$ standard errors.

$1,83 \mathrm{~m}^{2}$ pour la demi-lyre est,

$3,93 \mathrm{~m}^{2}$ pour la demi-lyre ouest sur laquelle des rameaux insérés relativement haut et retombant ont été laissés.

\section{a) Evolution du RG et de l'assimilation nette au cours du temps}

Elle est présentée sur la figure 8 pour les journées du 27 et du 28 août. On remarque l'allure à peu près symétrique et en phase avec le RG de la courbe correspondant à la demi-lyre est, alors que celle de la demilyre ouest présente un maximum nettement antérieur à celui du RG. Ce déphasage est à rapprocher de ce qus nous avons vu précédemment au sujet de l'éclairement de capteurs orientés nord-sud mais diversement inclinés : le maximum d'éclairement par le rayonnement direct, de la face supérieure du bras ouest se situe vers $7 \mathrm{~h} 30 \mathrm{TU}$, ce qui coïncide avec l'heure du maximum d'assimilation nette observée pour ce bras lors des 3 journées consécutives de mesure.

L'application de ce raisonnement au bras est conduirait à attendre un maximum relatif d'assimilation nette vers 16 h 30 solaire, ce qui ne semble pas confirmé par l'expérience. Il est probable que, là encore, apparaît la limite d'un mode de raisonnement uniquement fondé sur la captation de la lumière : même en 

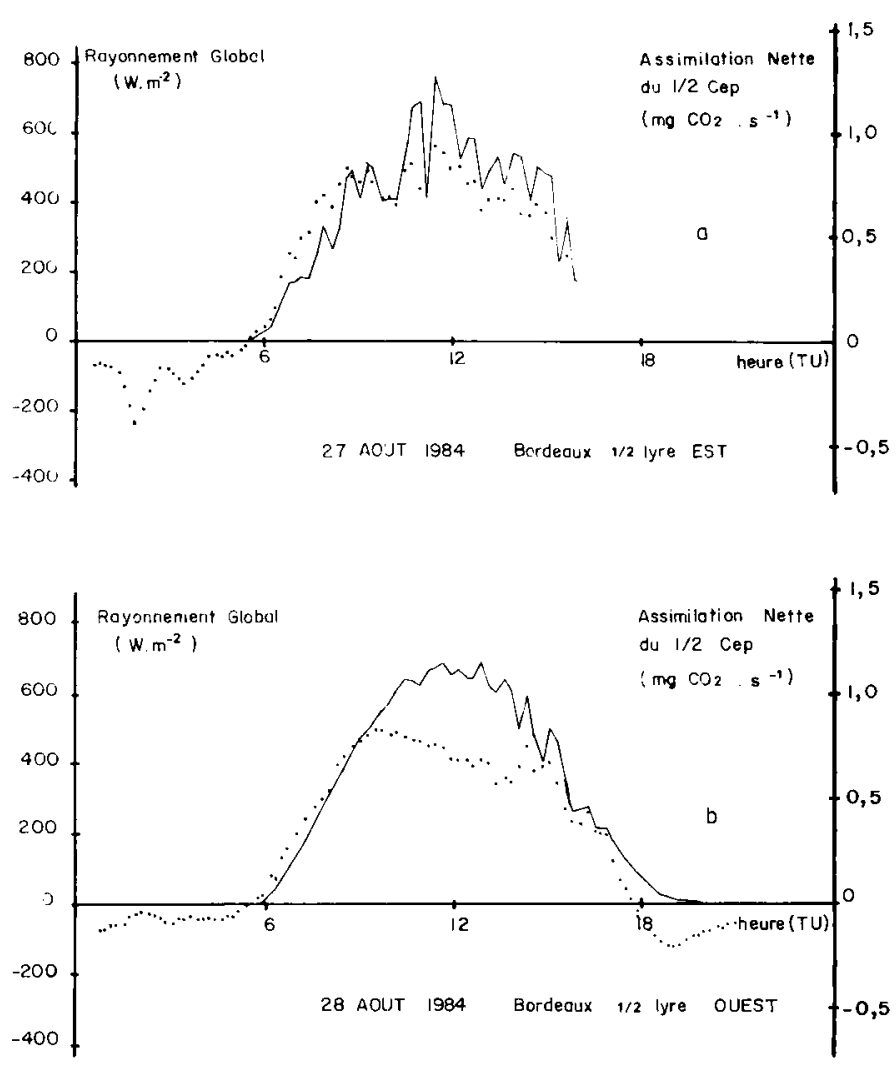

Figure 8

Evolution au cours de 2 journées consécutives du rayonnement global (__._. et de l'assimilation nette (.......) des demi-lyres est et ouest; Change over two consecutive days in total radiation $(\longrightarrow$ ) and net photosynthesis (.....) for East and West half-lyres.

a) journée du 27 août 1984,

b) journée du 28 août 1984 .

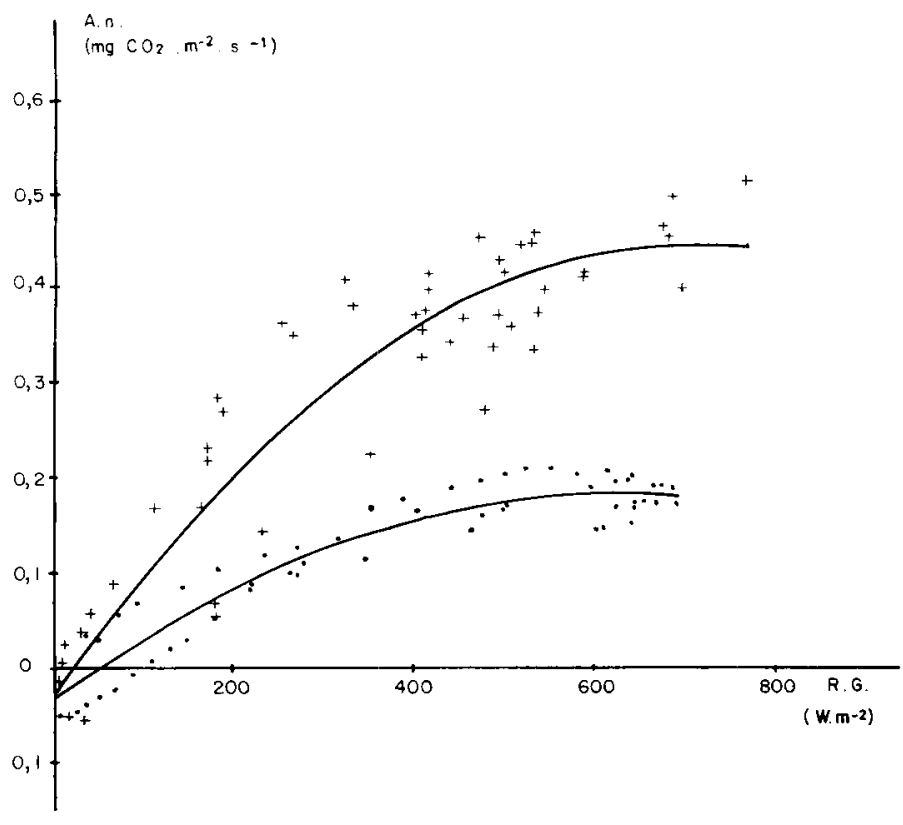

Figure 9

Assimilation nette par unité de surface foliaire en fonction du rayonnement global pour les 2 demi-ceps; Net photosynthesis per unit leaf area in relation to total radiation for the two half-plants.

+ demi-cep Est ; eastern half-plant,

- demi-cep Ouest; western half-plant. conditions hydriques non sévèrement limitantes, des facteurs externes, tels la température et l'hygrométrie atmosphériques ou internes comme une modification de l'état hydrique des tissus (mise en évidence par les mesures de potentiel foliaire, $\mathrm{cf}$. fig. 6 et 7) ou l'accumulation d'assimilats, conduisent à une réponse à la lumière significativement inférieure l'après-midi par rapport au matin.

\section{b) Relation assimilation nette-rayonnement global}

On montre figure 9 la réponse de l'assimilation nette au rayonnement global pour les 2 demi-lyres considérées. Le phénomène le plus remarquable est la réponse très inférieure de la demi-lyre ouest lorsque l'on considère l'assimilation nette par unité de surface foliaire. Nous n'avons guère d'éléments pour expliquer cette importante différence. Des phénomènes comme l'auto-ombrage (plus important dans le bras ouest qui présente un grand nombre de rameaux retombants et une surface foliaire sensiblement double de celle du bras est) ou une contrainte hydrique plus marquée, comme il apparaît dans la dynamique du potentiel hydrique foliaire, peuvent l'un et l'autre intervenir.

\section{DISCUSSION ET CONCLUSION}

\section{A. Relation entre assimilation nette et état hydrique}

Au cours des $1^{\text {res }}$ journées de mesure qui se situent en fin de période sèche, la photosynthèse nette est fortement limitée par la contrainte hydrique (cf. 19 août, fig. 2). La photosynthèse nette commence à diminuer lorsque la valeur du potentiel hydrique foliaire devient inférieure à $-1,1$ ou $-1,2 \mathrm{MPa}$; cette limitation est de plus en plus marquée au cours de la journée et elle s'accompagne d'une diminution de la conductance stomatique (fig. 3 ).

La période de réhumectation qui suit voit disparaître rapidement les effets majeurs de la contrainte hydrique sur l'assimilation nette. On peut cependant penser que la légère baisse de photosynthèse qui se manifeste, à rayonnement global donné, l'après-midi par rapport au matin (ex. les résultats des 22, 27 et 28 août), traduit une certaine difficulté de la plante à s'alimenter en eau. On constate d'ailleurs en même temps des potentiels hydriques foliaires de l'ordre de $-1,1 \mathrm{MPa}$.

\section{B. Relation entre comportement hydrique et éclaire- ment}

Les résultats présentés figures 6 et 7 font apparaître une différence dans le comportement hydrique des bras est et ouest ; en effet, la conductance stomatique maximale et le potentiel hydrique minimal sont plus faibles sur le bras ouest que sur le bras est. Une explication possible de ce comportement est liée au fait que, compte tenu de l'ombrage mutuel des 2 bras, l'énergie radiative absorbée par le bras est (faces supérieure + inférieure) est maximale le matin, c'est-à- 
dire à des heures où la demande climatique en eau est relativement faible et le déficit hydrique des tissus encore peu prononcé. Il en va différemment du bras ouest pour lequel l'éclairement maximal se produit l'après-midi et coïncide avec les heures les plus sèches et chaudes de la journée.

\section{Photosynthèse de 2 bras séparés en fonction de l'éclairement et de l'état hydrique}

Les résultats présentés font apparaître une différence de réponse à la lumière des bras est et ouest étudiés sur un même cep. L'assimilation nette par unité de surface foliaire semble environ 2 fois plus faible pour le bras ouest que pour le bras est. Plusieurs explications ont pu être évoquées :

- surface foliaire nettement plus importante du bras ouest, ce qui implique des phénomènes d'autoombrage accrus,

- dynamique de l'état hydrique et des résistances (stomatique et/ou interne) plus défavorable pour le fonctionnement du bras ouest.

Malgré des conclusions assez nettes dans certains domaines, une des limites importantes de ce travail tient à l'absence de référence radiative directement utilisable pour évaluer le P.A.R. (rayonnement actif pour la photosynthèse) absorbé par chaque bras.

Par suite de leur fiabilité douteuse certains jours, nous n'avons pas mentionné les résultats de transpiration mesurés à l'aide de la chambre CAT. Les quelques observations qui ont été faites allaient toutefois dans le même sens que les données de conductance stomati- que et de potentiel hydrique foliaire. Cette convergence de résultats indique que des mesures même ponctuelles et à l'échelle de la feuille peuvent donner des informations intéressantes pour évaluer l'état hydrique moyen d'une plante entière de la taille d'un cep de vigne. Des conclusions du même ordre pourraient être tirées de la comparaison entre mesures ponctuelles de photosynthèse (par le ${ }^{14} \mathrm{C}$ par exemple) et mesures globales. L'intérêt de mesures à l'aide d'une CAT de grandes dimensions réside évidemment dans la possibilité unique d'intégration (dans le temps et en considérant simultanément un très grand nombre de feuilles) qu'elle représente.

Enfin s'il est clair que l'on peut en attendre une meilleure compréhension du fonctionnement instantané de la plante et imaginer des études comparatives entre traitements permettant d'amorcer un raisonnement sur les mécanismes impliqués dans les différences constatées, il est tout aussi évident que le caractère ponctuel de telles études rend difficile la généralisation et l'obtention de conclusions fiables au niveau de l'élaboration complète du rendement; ils permettent cependant de proposer quelques hypothèses pour l'analyse future du comportement de la vigne.

Reçu le 21 février 1986. Accepté le 16 avril 1986.

\section{REMERCIEMENTS}

Les auteurs remercient A. Carbonneau (I.N.R.A., Bordeaux) responsable de l'ATP (Action Thématique Programmée) « Ecophysiologie de la vigne " pour l'aide qu'il leur a apportée dans le cadre de ces études.

\section{RÉFÉRENCES BIBLIOGRAPHIQUES}

Carbonneau A., 1976a. Principes et méthodes de mesure de la surface foliaire. Essais de caractérisation des types de feuilles dans le genre Vitis. Ann. Amélior. Plantes, 26 (2), 327-343.

Carbonneau A., 1976b. Analyse de la croissance des feuilles du sarment de vigne: estimation de sa surface foliaire par échantillonnage. Connaiss. Vigne Vin, 10 (2), 141-159.

Carbonneau A., 1980. Recherche sur les systèmes de conduite de la vigne : essai de maîtrise du microclimat de la plante entière pour produire économiquement du raisin de qualité. Thèse Doct. Ing., Univ. Bordeaux II, $235 \mathrm{p}$

Carbonneau A., Loth C. de, 1985. Influence du régime d'éclairement journalier sur la résistance stomatique et la photosynthèse brute chez Vitis vinifera L. Cv « Cabernet-Sauvignon". Agronomie, 5, 631-638.

Daudet F. A., 1986. Système simple pour mesurer en continu in situ les échanges gazeux de surfaces végétales de plusieurs mètres carrés d'extension. (En préparation.)

Foex G., 1895. Cours complet de Viticulture. Ed. Coulet, Montpellier ; Masson, Paris, $1120 \mathrm{p}$.

Katerji N., Carbonneau A., Delas J., 1986. Etude du fonctionnement hydrique et photosynthétique de deux systèmes de conduite de la vigne au cours d'un cycle de dessèchement édaphique. Comm. $3^{e}$ Symp. Int. "Physiologie de la vigne », 24-27 juin, Bordeaux (France), 6 p.

Kliewer W. M., Lider L. A., 1970. Effects of day temperature and light intensity on growth and composition of Vitis vinifera L. fruits. J. Am. Soc. Hortic. Sci., 95, 766-769.

Kriedemann P. E., 1968. Photosynthesis in vine leaves as a function of light intensity, temperature and leaf age. Vitis, 7, 213-220.
Kriedemann P. E., Kliewer W. H., Harris J. M., 1970. Leaf age and photosynthesis in Vitis vinifera L. Vitis, 9, 97-104.

Kriedemann P. E., Torokfalvy E., Smart R. E., 1973. Natural occurence and photosynthetic utilisation of sunflecks by grapevine leaves. Photosynthetica, 7, 18-27.

Millar A. A., 1972. Thermal regime of grapevines. Am. J. Enol. Vitic., 23, 173-176.

Ravaz L., 1909. Influence des opérations culturales sur la végétation et la production de la vigne. Ann. Ec. Nat. Agric. Montpellier, 8, 231-291.

Ribereau-Gayon G., 1966. Etude du métabolisme des glucides, des acides organiques et des acides aminés chez Vitis vinifera $L$. Thèse, série $\mathrm{A}, \mathrm{n}^{\circ} 4712$ (cité par Carbonneau, 1980).

Scholander P. F., Hammel H. T., Bradstreet E. D., Hemmingsen E. A., 1965. Sap pressure in vascular plants. Sci. $N y$., 148, 339-346. Seguin G., 1970. Les sols de vignobles du Haut-Médoc. Influence sur l'alimentation en eau de la vigne et sur la maturation du raisin. Thèse d'Etat, Univ. Bordeaux, $141 \mathrm{p}$.

Shaulis N. J., Amberg H., Growe D., 1966. Response of concord grapes to light, exposure and geneva double curtain training. Proc. Am. Soc. Hortic. Sci., 89, 268-280.

Smart R. E., 1973. Sunlight interception by vineyards. Am. J Enol. Vitic., 24, 141-147.

Smart R. E., 1976. Implications of the radiation microclimate for productivity of vineyards. Ph. D. Thesis, Cornell Univ., Ithaca, $\mathrm{NY}, 174 \mathrm{p}$.

Tse-Horng Liu W., 1974. Soil plant water relations in a New York vineyard. $\mathrm{Ph}$. D. Thesis, Cornell Univ., Ithaca, NY, $145 \mathrm{p}$. 